- the introduction of strategic napping

- purchase of flat bed chairs to facilitate same

- environmental - a dedicated nap station 'Snoozzone' proposed

- education programme on fatigue \& fatigue mitigation strategies introduced

- Fatigue risk scan is being completed and data collection is taking place

The fatigue scan is expected to influence the organisation of work and work scheduling to minimise the impact of fatigue and to provide adequate rest periods. The final FRMS (Fatigue Risk Management Strategy) document has now been completion.

Discussion Recent research shows that one in four doctors report driving home following work shifts when fatigued. There have been a number of deaths of doctors involved in road traffic accidents following night shifts in the UK in recent years associated with fatigue. Following the very positive feedback from staff following the successful introduction of a FRMS in a children's hospital, a programme to introduce FRMS is now being extended to public hospitals throughout Ireland. This will not only help to improve the health and safety of doctors and other healthcare workers but shall ultimately improve patient care. The FRMS shall be launched nationally later in 2017 in liaison with WHWU (Workplace Health and Wellbeing Unit), HSE to address fatigue in public funded hospitals in Ireland. A position paper is currently being written by the authors for Faculty of Occupational Medicine Ireland.

\section{EPIDEMIOLOGY IN THE WAITING ROOM: CAN THE OCCUPATIONAL PHYSICIAN DEMONSTRATE THE ASSOCIATION BETWEEN WORK-RELATED STRESS AND METABOLIC SYNDROME?}

${ }^{1}$ Nicola Magnavita* ${ }^{1}$ Ilaria Capitanelli, ${ }^{1}$ Erika Alessandra Lops, ${ }^{1}$ Sara Manetta, 2,3 Sergio Garbarino. 'Institute of Public Health, Università Cattolica del Sacro Cuore, Rome, Italy; ${ }^{2}$ Department of Health Sciences, University of Genoa, Genoa, Italy; ${ }^{3}$ Department of Neuroscience, Rehabilitation, Ophthalmology, Genetics and Maternal/Child Sciences (DINOGMI), University of Genoa, Genoa, Italy

\subsection{6/oemed-2018-ICOHabstracts. 1626}

Introduction Work-related stress (WS) has been proposed as a risk factor for the development of the metabolic syndrome (MetS). The number of studies, however, is limited, and results are conflicting. The occupational health physician can give an effective contribute to clarify this point by systematically collecting data from workers waiting for medical examination.

Methods During 2016, the workers were invited to complete a questionnaire that included the Effort-Reward Imbalance scale (ERI) of Siegrist and the Support scale from the DCS model of Karasek, before their medical examination in the workplace. 1904 persons (male 32.6\%, female 67.4\%) participated in the study. The mean age was 47.35 \pm 9.38 . Health data were obtained from medical surveillance records.

Results The prevalence of workers with high blood pressure (316, 16.6\%), high cholesterol/reduced HDL-cholesterol (511, 26.8\%), high triglycerides (195, 10.2\%), high blood glucose
(91, 4.8\%), and obesity $(677,35.6 \%)$ led to a diagnosis of MetS in 196 workers (10.3\%). In univariate logistic regression analysis, WS was significantly associated with the occurrence of MetS (OR 1.82; 95\% CI: 1.41 to 2.35). ERI was also significantly associated with hypertriglyceridemia (OR 1.64; 95\% CI: 1.27 to 2.13) and with overweight (OR 1.33; 95\% CI: 1.11 to 1.59 ). The association was still significant in multivariate models, after correction for confounders.

Discussion The observed association between WS and MetS deserves particular attention. The root causes of stress in workers must be investigated so as to have information for prevention.

\section{HEALTH AND WELLBEING- WORK-LIFE IMBALANCE IN DEVELOPING COUNTRIES}

Janice Green. JN Bank Limited, Kingston, Jamaica

10.1136/oemed-2018-ICOHabstracts. 1627

Introduction The concept of work-life balance, health and well-being is about behaviour change management in ones physical, social, and mental state to attain a measure of stability. This stability can be achieved through creative and substantial preventative and or corrective actions taken in a collaborative manner with other relevant persons.

Professors Jodi Oakman and Siew Chan in their safety journal on the topic Risk management: Where should we target strategies to reduce work-related musculoskeletal disorder; did a study on Australian companies with high reported instances of work related musculoskeletal disorders (WMSDs). In this study, it was found that there was evidence to support the claim that there is a link between WMSD and psychosocial factors. Psychosocial factors such as stress, work place demand, job security and working hours are said to be predicators of WMSDs. The study also revealed that there is an inverse relationship between work-life balance and psychosocial factors. A low level of work-life balance indicates that there is a high level of stress, workplace demand complicated by work-related musculoskeletal disorders.

It's a general belief that the mean age for employees at the reproductive age is 35 years. It is at this crossroads that most employees are working the hardest at balancing their priorities; work, family, social and their personal development. It is at this juncture of their lives that the psychosocial factors of work related stress, economic stress, physical inactivity and life style illnesses take centre stage. The imbalance occurs as one or two priorities are given more attention and the focus on the others significantly decreases.

From the premise of a proactive employer who is concerned about the health and wellbeing of the workers; behaviour change intervention programs become necessary. These interventions are relevant to ensure that workers remain healthy so that productivity does not suffer and the worker will benefit, eventually with financial rewards. The worker more often is sometimes is not able to individually, and unilaterally, restore the require work-life balance, hence the support of the employer becomes important.

Methods To achieve this balance there has to be a vision of what the behaviour change intervention programme is to achieve. How 
will the program impact lifestyle and lay the foundation for good health, a decent quality of life and wellbeing.

From the vision, the mission will be derived as to the targets; how they will they be measured and over what time period. The values of the program has to be shared where workers and the employer agree and work together to achieve common goals.

To strategically attain work life balance; a principled health and wellness programs has to be developed and integrated into the business operations as a key performance indicator. By so doing the programs will be aligned to the business strategies.

Results With this alignment some of the possible results that could be realised are:

- A culture of safety

- Reduction in on the job accidents

- Increased productivity and reduced absenteeism

- Reduction in sick days leading to reduction in utilisation of health insurance scheme

- Improved profit margins

- Improved brand image - employer of choice

- A more satisfied employee

- A changed worker with improved attitude and behaviour

Discussion Many blue chip companies in developed countries have established programs in place to achieve work-life balance, health and wellbeing for their staff. Some of these companies are Google, Alcoa, Fiat and Virgin Airlines, to name a few well know ones. Paul O'Neill, former CEO of Alcoa in a CNN May 2014 interview spoke about safety as a priority at Alcoa. It could be gleaned from his remarks that work life balance was achieved from the involvement of management and their commitment to a vision. Also that the desired values and attitudes for a culture of safety, work-life balance, health and wellbeing were cascaded down to the workers from the strategic level and were accepted by the employees.

In summary, Work-Life Balance Health and Well-Being is not an insurmountable goal. To support the achievement of this balance for workers, the ILO under its pillar of Decent Work has a health and safety convention that serves as an international standard for nations to pattern in their national programs. In this respect, legislations have been passed in many countries in support of the Convention. It is of note that European countries in support of the promotion of workers health and wellbeing have passed legislations. The European Framework Agreement on work-related stress was one such legislation that was passed on 8 October 2004 .

There is also the Italian Regulatory Framework for Health and Safety in the Workplace, Legislative Decree 81/08. This was bench marked form the UK Health and Safety Management Standard for Work related Stress, Belgian Screening, Observation, Analysis, Expertise (SOBANE) Strategy of Risk Management and German stress-psychology-health (start).

The principle of work of work-life balance is generally accepted globally. However there is a perception of institutional inertia in many developing countries to enact the necessary legislations to support a national policy and program framework for occupational health and safety. It is hoped that developed countries that are signatories to the ILO Occupational Health and Safety Convention as well as countries in the European Unions, will extend more significant and substantial support in the areas of human resource and capacity building, as well as financial assistance to help developing countries strike that desired balance.
225

A ROLE FOCUSED SELF-HELP COURSE REDUCES STRESS AND SUBSEQUENT WORK FUNCTIONING PROBLEMS IN WORKERS WHO HAVE INFORMAL CARE RESPONSIBILITIES

EJ Boezeman, K Nieuwenhuijsen, JK Sluiter. Academic Medical Centre, University of Amsterdam, Department: Coronel Institute of Occupational Health, Amsterdam Public Health research institute, Amsterdam, The Netherlands

\subsection{6/oemed-2018-ICOHabstracts. 1628}

Introduction Many paid workers who fulfil informal care responsibilities in their private situation report stress complaints and impaired functioning at work. It was examined whether a newly developed role-focused self-help intervention decreases caregiver stress and distress, and work functioning problems, in these workers.

Methods A study that had a randomised controlled design (intervention vs wait-list control) was conducted. Workers suffering stress due to involvement in informal care activities were participants $(n=128)$. The workers allocated to the intervention group received the role-focused self-help course. The workers allocated to the control group received the intervention after all measurements. Caregiver stress (primary outcome), distress, work functioning, care-to-work interference, and care-to-social and personal life interference, were measured prior to the random allocation (pre-test) and one (posttest 1) and two (post-test 2) months after allocation. Mixed Model ANOVAs and mediation analyses were used in dataanalysis.

Results The mixed model ANOVAs showed that two months after allocation the workers allocated to the intervention group had decreased levels of caregiver stress and distress as compared to the workers allocated to the no treatment control group. Further, the mediation analysis suggested that involvement in the intervention decreases functioning problems in workers who have informal care responsibilities because it reduces caregiver stress.

Discussion The role-focused self-help course intervention decreases levels of caregiver stress and distress in workers who suffer stress due to involvement in informal care activities. Because of this effect, the intervention also plays a role in the work functioning of these types of workers. The intervention represents a contribution to the literature and a means for occupational health professionals and organisations to contribute to the health and functioning of workers who combine paid work with informal care activities

\section{HIGH BURNOUT LEVEL AS MAIN DETERMINANT OF LOW WORK ABILITY AMONG FLIGHT ATTENDANTS}

${ }^{1,2}{ }^{2} \mathrm{M}$ Milošević* ${ }^{1}, 1 \mathrm{~J}$ Mustajbegović, ${ }^{2} \mathrm{~T}$ Madžar, ${ }^{3} \mathrm{M}$ Bubas, ${ }^{4} \mathrm{~T}$ Rogina, ${ }^{5} \mathrm{~T}$ Furlan. ${ }^{1}$ University of Zagreb, School of Medicine, WHO Collaborative Centre for Occupational Health, Croatia; 'University of Zagreb, School of Medicine, Centre for Sports Medicine and Health Promotion at Work, Croatia; ${ }^{3}$ Croatian Institute for Health Protection and Safety at Work, Zagreb, Croatia; ${ }^{4}$ Varaždin County Heath Care Centre, Varaždin, Croatia; ${ }^{5}$ Adriamedic Clinic for Occupational Medicine, Pazin, Croatia

\subsection{6/oemed-2018-ICOHabstracts. 1629}

Introduction The roles of flight attendants are to provide excellent customer service to passengers while ensuring their comfort and safety throughout the flight. Flight attendants have more responsibilities than most front-line employees in the service industry, as they are first trained to maintain cabin 